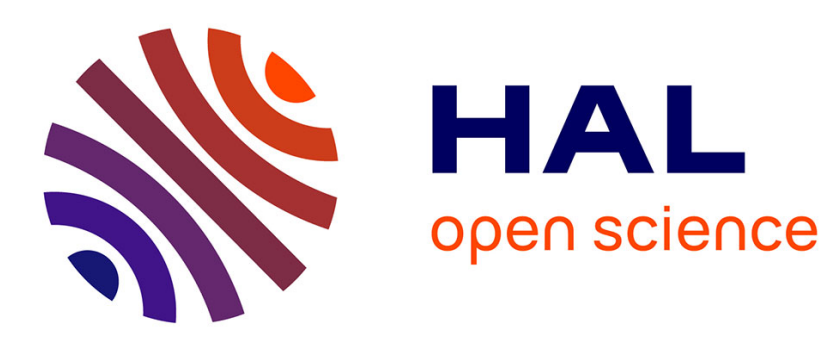

\title{
Stratégies pour de nouveaux progrès techniques et économiques en aviculture
}

\author{
B. Sauveur
}

\section{To cite this version:}

B. Sauveur. Stratégies pour de nouveaux progrès techniques et économiques en aviculture. Productions Animales, 1991, 4 (1), pp.31-40. hal-00895922

\section{HAL Id: hal-00895922 \\ https://hal.science/hal-00895922}

Submitted on 1 Jan 1991

HAL is a multi-disciplinary open access archive for the deposit and dissemination of scientific research documents, whether they are published or not. The documents may come from teaching and research institutions in France or abroad, or from public or private research centers.
L'archive ouverte pluridisciplinaire HAL, est destinée au dépôt et à la diffusion de documents scientifiques de niveau recherche, publiés ou non, émanant des établissements d'enseignement et de recherche français ou étrangers, des laboratoires publics ou privés. 
INRA Prod. Anim., 1991, 4 (1), 31 - 40

\section{B. SAUVEUR}

\section{INRA}

Station de Recherches Avicoles 37380 Nouzilly
Stratégies pour de nouveaux progrès techniques et économiques en aviculture

\section{Les viandes de volailles connaissent depuis vingt ans une progression enviée : produites aujourd'hui avec une technicité élevée, elles satisfont de nombreux aspects de la demande des consommateurs. Des possibilités de progrès existent cependant encore. De nouvelles stratégies scientifiques (apports de la génétique quantitative et moléculaire, de la nutrition, des techniques de reproduction ...) et structurelles (organisation de la production, élaboration des produits ...) devraient permettre une diffusion encore plus large des produits avicoles futurs.}

\section{Résumé}

La réunion d'arguments scientifiques, techniques et économiques peut permettre d'élaborer encore de nombreuses stratégies de progrès pour l'aviculture. Ainsi, les améliorations à attendre de la génétique quantitative classique sont loin d'être terminées. Des critères nouveaux (anatomiques ou physiologiques) de sélection sont en préparation et ils pourront être complétés par des études de polymorphismes d'ADN utilisés comme indicateurs de caractères économiques, notamment de résistance à certaines maladies. Ces méthodes sont cependant coûteuses, de même que la transgénèse qui n'en est encore, quant à elle, qu'au stade des études méthodologiques. Elles ne seront donc mises en place que si la possibilité d'amélioration d'un critère économique important est démontrée. Dans l'entre temps, toute modification de la croissance par administration de somatotropine paraît exclue.

L'utilisation métabolique des matières premières alimentaires devra de son côté être de mieux en mieux connue et quelquefois accrue par utilisation d'enzymes afin de réduire les rejets dans l'environnement (azote et phosphore). L'obtention d'aliments bactériologiquement irréprochables devra aussi être assurée. L'avenir de la reproduction est fortement lié à la maîtrise de la consommation alimentaire des animaux reproducteurs et, ultérieurement, à l'usage généralisé de l'insémination artificielle qui permettra une gestion totalement séparée des deux sexes.

La pathologie se diversifie avec l'apparition de syndromes complexes (anémie infectieuse, troubles locomoteurs ...) tandis que le portage sain de bactéries pathogènes pour l'homme constitue désormais l'autre source principale de préoccupations. Les techniques de recombinaison génique devraient permettre, en juxtaposant les gènes de différents épitopes à l'intérieur d'une même bactérie ou virus, la création de vaccins multipotents qui, autre innovation, seront peut-être administrés in ovo à l'embryon.

Les modes d'élevage ont encore à s'adapter aux spécificités des pays (et même des régions) en privilégiant toujours la qualité, notamment microbiologique, des produits obtenus (volailles et oeufs) et les problèmes de bien-être animal. Les produits avicoles eux-mêmes seront de plus en plus souvent l'objet d'une première, voire d'une seconde transformation améliorant sans cesse leur commodité d'emploi et leurs caractéristiques diététiques ; ils seront valorisés par une politique de marque et d'" image-qualité ». Globalement, la gestion par résultat économique vrai intégrant l'ensemble d'une filière est probablement appelée à se développer.
Grâce aux progressions remarquables de volume qu'elles ont connues au cours des trois dernières décennies, les viandes de volaille produites en France représentent aujourd'hui le même tonnage que chacune des viandes bovine et porcine. Cette évolution est certainement loin d'être achevée puisqu'on prévoit à l'échelon mondial un doublement de la production avicole entre 1986 et l'an 2000 et, dans la seule CEE, une augmentation de $+38 \%$.

Cette évolution s'est accompagnée, surtout depuis 1988, d'une spectaculaire concentration des moyens de production. Aujourd'hui $58 \%$ du tonnage français de viande de volaille sont produits par 10 groupes industriels et $36 \%$ par 3 seulement d'entre eux. Cette concentration et le développement de liaisons verticales de plus en plus étroites au sein des filières de production devraient permettre à l'Aviculture de réfléchir à des stratégies de progrès nouvelles dont certains éléments, tant scientifiques ou techniques qu'économiques sont présentés ici.

\section{1 / Efficacité de la génétique quantitative}

\section{1 / Le progrès génétique n'est pas mort !}

Contrairement à ce qui est affirmé quelquefois, il n'existe aucune preuve d'un plateau dans le gain génétique des performances de croissance des poulets de chair. Les coefficients d'héritabilité continuent à être de grandeur moyenne et les progrès enregistrés à chaque 
Tableau 1. Estimations des gains génétiques annuels sur différents critères de croissance du poulet de chair.

\begin{tabular}{|l|c|c|}
\hline \multicolumn{1}{|c|}{ Société } & ISA (1987) & Arbor Acres (1989) \\
\hline Croissance $(\mathrm{g})$ & +45 & +40 \\
\hline Age à l'abattage (j) & $-0,8$ & $-0,4$ \\
\hline Indice de consommation (g aliment/g poids vif) & $-0,03$ & $-0,02$ \\
\hline Rendement en viande (\% du poids vif) & $+0,25$ & $+0,20$ \\
\hline
\end{tabular}

génération montrent que la variabilité génétique contribuant à augmenter le poids corporel est loin d'être épuisée. Une estimation du gain génétique annuel effectuée par deux sociétés de sélection différentes est donnée, dans le t́ableau 1, à titre d'exemple. Les nouvelles méthodes de calcul (BLUP, modèle animal) qu'ont développé les généticiens des grosses espèces animales n'apporteront pas de révolution à la sélection avicole mais contribueront, en permettant une prise en compte plus complète des parentés, à la poursuite du progrès.

\section{2 / Prise en compte de nouveaux critères de sélection}

Dès 1979, Van der Eyden prévoyait la nécessité de prendre en compte des caractères tels que la teneur en graisse des carcasses, la résistance du squelette, la réponse au stress ou la part de filets sur la carcasse. La création de génotypes maigres est un bon exemple où l'introduction de critères de sélection nouveaux, mais simples, a permis une évolution décisive du produit (Leclercq 1989). Elle aboutit en effet à un rendement accru en viande consommable des carcasses, à une augmentation de l'efficacité alimentaire d'environ $5 \%$, et de l'efficacité protéique de $3 \%$ avec pour conséquence une économie de protéines et une diminution de rejet d'azote dans l'environnement. Un autre pas décisif dans la progression des rendements en viande sera constitué dans un avenir proche par l'usage de mesures physiques (tomographie à ultra-sons et peut-être conductibilité totale) qui permettront d'apprécier directement la valeur des candidats à la sélection plutôt que de devoir s'adresser à leurs collatéraux.

D'autres caractères doivent aussi être pris en compte de façon urgente, à savoir :

- dans les lignées lourdes, la fertilité des mâles reproducteurs du fait de l'existence de corrélations génétiques négatives entre ce critère et la vitesse de croissance. Sans sélection, on risquerait de connaître d'ici 10 ans une baisse moyenne de fertilité proche de $5 \%$ (figure 1). - dans les lignées de poules pondeuses, des caractères de qualité de l'oeuf tels que l'homogénéité de coloration de la coquille, la structure physique de l'albumen ou l'existence d'inclusions, tous critères qui participent à des degrés divers à l'acceptabilité du produit.

Des étapes suivantes de sélection pourraient être la prise en compte de critères réellement nouveaux tels que l'intensité de réponse immunitaire après vaccination, caractère dont l'héritabilité paraît comprise entre 0,06 et 0,53
(Cheng et Lamont 1990) ou bien, dans les lignées à croissance rapide, les taux d'IGF1 circulants qui semblent pouvoir en constituer un éventuel critère de prédiction.

La résistance génétique à la chaleur estimée par les performances de croissance en environnement chaud ne s'avère pas être un caractère facile à sélectionner (Picard 1990, communication personnelle). A l'inverse, la sélection sur des critères sociaux d'adaptation à l'élevage en groupes a été réalisée à la Station de Recherches Avicoles de l'INRA (Mills et Faure 1991) et constitue un modèle intéressant de ce qui pourrait être développé dans le futur.

\section{2 / Les promesses de la génétique moléculaire}

\section{1 / Utilisation de gènes majeurs}

A côté du jugement global des effets d'un grand nombre de gènes que constitue la génétique quantitative, l'exploitation de gènes majeurs pour lesquels sont connues des mutations affectant des caractères économiques est

Figure 1. Prévision du progrès génétique concernant le poids vif des poulets âgés de 6 semaines et le taux de fertilisation des oeufs entre 1990 et 2000. L'évolution de la fertilité est indiquée dans deux hypothèses: avec et sans prise en compte de ce critère dans les index de sélection (Reddy et Sadjardi 1990).

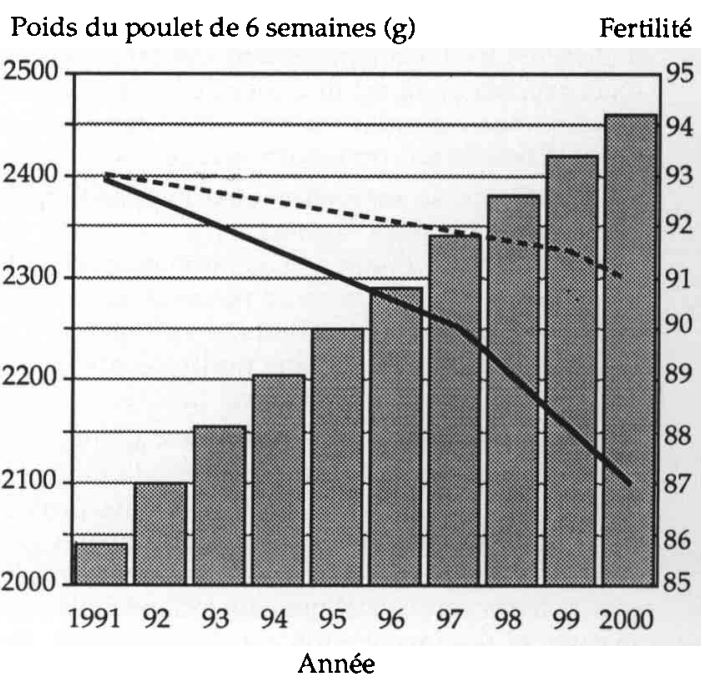

Fertilité : — sans sélection ------avec sélection 
une pratique déjà ancienne : exemples du gène récessif de nanisme dw, des gènes d'emplumement permettant l'autosexabilité ou du gène " cou nu " très utilisé chez le poulet " Label ».

L'identification plus récente de nouvelles liaisons entre des critères de production et certaines parties du génome, nommément le Complexe Majeur d'Histocompatibilité (CMH) et des gènes viraux endogènes, est tout à fait prometteuse. Les gènes du CMH sont les plus connus et on sait par exemple que les populations portant l'allèle $\mathrm{B} 21$ sont résistantes à la maladie de Marek alors que l'haplotype B1 est le plus déficient dans la production d'anticorps contre Salmonella pullorum (Nordskog 1983). Le mode d'action des gènes viraux endogènes (ev) reliés au virus de la leucose est encore mal connu; une des découvertes les plus intéressantes a été la démonstration d'une liaison très étroite, sur le chromosome sexuel mâle, entre ev 21 et la mutation d'emplumement lent, expliquant chez les animaux porteurs de cette dernière une plus grande sensibilité à la leucose. De même, le gène ev 12 serait associé au taux d'ovulation et même au poids de coquille (Kuhlein et Zadworny 1990).

\section{2 / Etude des polymorphismes d'ADN}

Dans la situation très répandue où l'on ne connaît pas de gènes directement responsables d'un caractère quantitatif, on est conduit à rechercher plutôt les liaisons existant de façon statistique entre ce caractère et certains gènes marqueurs. Les progrès réalisés en biochimie moléculaire permettent de rechercher aujourd'hui des polymorphismes de l'ADN et non plus seulement ceux des protéines synthétisées. De tels polymorphismes d'ADN sont révélés par exemple par les techniques dites de RFLP (" polymorphisme de longueur de fragments de restriction" mis en évidence par électrophorèse des fragments après usage d'enzymes de restriction). Lorsqu'une association statistique est démontrée avec un caractère quantitatif, on donne à la zone d'ADN correspondante l'appellation de Q.T.L. (Quantitative Trait Loci) et, si son effet est suffisamment fort, ce Q.T.L. peut être traité comme un gène majeur (Mallard 1990).

Les avantages potentiels promis par ces approches sont énormes puisqu'elles devraient permettre, une fois les cartes de liaison établies :

- d'identifier les génotypes «supérieurs» sans avoir à mesurer systématiquement les performances zootechniques ;

- de sélectionner la plupart des caractères chez les deux sexes, y compris chez celui où il ne s'exprime pas ;

- d'évaluer très tôt la valeur d'un individu pour un caractère qui ne's'exprime que tardivement, etc. (Siegel 1990)

Néanmoins, l'établissement de ces liaisons est aujourd'hui encore extrêmement coûteux et il n'est donc pas démontré qu'il pourra être amorti dans le contexte d'une sélection commerciale. Par ailleurs et surtout, les liaisons ainsi établies risquent de ne pas être retrouvées de façon stable d'une génération à la suivante.

\section{3 / Transfert de gènes}

Différentes stratégies de transfert sont aujourd'hui envisageables :

- l'usage de vecteurs rétro-viraux rendus non pathogènes et utilisés en début de développement embryonnaire a été le plus envisagé chez les volailles car ces vecteurs peuvent assurer la diffusion du gène introduit jusque dans la lignée germinale. Ils présentent cependant plusieurs inconvénients liés en particulier au risque de recombinaison avec un gène viral endogène du poulet qui redonnerait au virus un pouvoir pathogène.

- l'infection de cellules germinales primordiales par un rétrovirus et leur ré-introduction dans le système circulatoire embryonnaire autour de 48 h est une variante améliorée de la technique précédente.

- la micro-injection d'ADN dans le disque germinal, bien que difficile, a été réalisée et suivie d'un développement embryonnaire dans une coquille étrangère. Cependant, des pertes progressives des séquences étrangères d'ADN introduites semblent intervenir.

- l'insertion de gènes étrangers dans des spermatozoïdes soit in vitro, soit in vivo (par voie intratesticulaire) et leur transmission à la descendance est également une voie qui mérite d'être étudiée.

Si le choix de la méthode de transfert ne va donc pas de soi, celui des gènes à transférer reste aussi très ouvert à discussion. Ainsi Bosselman et al (1990) ont réussi chez le poulet un transfert de gène de GH (hormone de croissance) induisant une élévation du taux circulant d'hormone, mais on sait que l'apport de $\mathrm{GH}$ exogène ne modifie pas la croissance du poulet (cf. plus loin). On dispose de même de plusieurs des gènes codant pour les protéines de l'oeuf, mais aucune application n'est actuellement envisageable par défaut de connaissance des mécanismes physico-chimiques par lesquels ces protéines interviennent dans la qualité de l'oeuf (Bulfield et Mc Kay 1987).

C'est donc certainement la transmission d'un facteur de résistance à une maladie qui risque d'être la première application de la transgenèse chez les oiseaux. Il pourrait s'agir soit d'un gène de résistance proprement dit, soit d'un gène de l'agent pathogène ; Freeman et Bumstead (1987) ont ainsi proposé le transfert du gène env du virus de la leucose lymphoïde dont la protéine induite, en saturant les récepteurs cellulaires de l'hôte, empêcherait l'adhésion des particules virales exogènes.

A plus long terme, on peut imaginer de modifier des caractéristiques métaboliques; ainsi l'absence d'enzyme(s) rendant indispensables certains acides aminés, pourrait être surmontée par le transfert du ou des gènes correspondant(s) (par exemple la $\beta$ asparto-kinase; Larbier 1986, communication personnelle). Certains des gènes codant pour les enzymes de la lipogenèse hépatique (Acetyl CoA-carboxylase par exemple) pourraient également, selon Mallard (1990), faire l'objet d'une transfection.

Compte-tenu du coût élevé et de la complexité (au moins actuels) des opérations de génie génétique, il est clair que, de toutes 
façons, seuls certains gènes à action réellement déterminante seront manipulés en pratique et ce, chez un nombre faible d'animaux constituant un noyau à partir duquel des schémas classiques de rétro-croisement permettront la diffusion du progrès obtenu.

\section{3 / Modifications physiologiques de la croissance}

Contrairement à ce qui a été décrit chez plusieurs espèces de mammifères, aucune accélération de la croissance ne peut être obtenue chez le poulet par simple administration d'hormone de croissance, même homologue. De même, les peptides hypothalamiques contrôlant la sécrétion de GH sont sans effet. Probablement ce défaut de réponse est-il attribuable au non respect de la pulsatilité naturelle de la décharge de GH dont l'amplitude et la fréquence des pics varie avec l'âge et le sexe (Johnson 1989). On pourrait par ailleurs s'intéresser à l'administration d'IGF ou de différents facteurs de division cellulaire (FGF, EGF ...) ou bien provoquer des réponses immunitaires contre la somatostatine.

On se souvient cependant avec inquiétude des dégâts causés il y a 30 ans par l'utilisation d' « hormones » stéroïdiennes dans l'élevage du poulet. Ceci impose une grande circonspection avant de revenir à des interventions de type hormonal, même très éloignées des précédentes, le consommateur ne semblant pas prêt à faire la distinction. La même réticence devrait s'appliquer à l'usage des $\beta$-agonistes dont les effets sont faibles et discutés chez les volailles.

\section{4 / De nouvelles stratégies alimentaires sont-elles encore possibles?}

Si la connaissance des besoins alimentaires des volailles est aujourd'hui satisfaisante, des progrès restent réalisables grâce à une meilleure connaissance des matières premières, des traitements à leur appliquer et de leur utilisation par les animaux. De nouvelles stratégies d'alimentation peuvent en résulter.

\section{1 / Les matières premières et leurs traitements}

Au-delà de la composition chimique classique, on exige maintenant de connaître la fraction digestible, voire "métabolisable» ou " disponible» de chaque nutriment contenu dans une matière première et ce à l'état natif ou après divers traitements physiques (broyage, cuisson, extraction, extrusion, etc...) ou enzymatiques. L'exemple des farines de viandes montre aussi que la digestibilité d'un nutriment (ici les graisses saturées) peut diminuer lorsque le taux d'incorporation dans un aliment composé augmente. Enfin, des critères analytiques nouveaux peuvent venir s'ajouter, tels que les teneurs en facteurs anti-nutritionnels de tous genres (anti-enzymes par exemple, ou facteurs complexants), en enzymes naturels (phytases), voire en toxines dans les matières premières les moins connues. Le nombre d'études à réaliser en ce domaine reste donc considérable.

Une nouvelle demande apparaît aussi aujourd'hui pour des aliments composés bactériologiquement sains; le procédé dit APC (Anaerobic Pasteurising Conditioning) permettant d'augmenter la température de la farine sans qu'elle ne devienne trop humide offre de ce point de vue des perspectives intéressantes.

\section{2 / Stratégies alimentaires}

Les années 80 auront marqué, en aviculture, la fin des mélanges alimentaires simplifiés de type maïs-tourteau de soja. Pour des raisons économiques, les «formules " de demain utiliseront un grand nombre de sous-produits des industries alimentaires ou non (distillerie, amidonnerie, huilerie), soit sous leur forme brute soit après transformation par des enzymes ou des micro-organismes (Blum 1990).

Les soucis de protection de l'environnement conduisent à rechercher une diminution des rejets, notamment azotés et, en conséquence, à promouvoir la notion de protéine alimentaire " idéale " dont la composition en acides aminés serait la mieux adaptée possible aux besoins des animaux. Ceci peut favoriser le développement de nouveaux acides aminés de synthèse ou issus de fermentations, notamment du tryptophane et surtout de la thréonine dont la production à partir d'E. coli modifié est actuellement à l'essai pour un marché français estimé aux environs de 2000 tonnes (soit la moitié de celui de la lysine).

Des stratégies alimentaires quasiment opposées peuvent être envisagées lorsque la concentration géographique des élevages est faible. En aviculture tropicale par exemple on pense non seulement utiliser les matières premières originales (après détoxification) mais également les incorporer dans des mélanges granulés de faible densité énergétique. Ceux-ci contiennent forcément une fraction indigestible élevée, mais offrent aux poules une meilleure régulation de leur ingéré et, partant, des performances plus élevées en présence de stress thermiques (Picard 1990). L'usage d'aliments à composition variable au cours de la journée et de systèmes dits de libre-choix (apport de calcium séparé par exemple) mérite également d'être développé pour les poules pondeuses (Sauveur et Clavreul 1984).

\section{5 / La reproduction des espèces avicoles n'est pas optimale}

La prolificité des espèces de volailles destinées à la production de viande (poules lourdes, dindes, canes de Barbarie) est encore limitée par de nombreux facteurs tels que :

- Une maturité sexuelle relativement tardive (5 à 10 semaines de plus que chez la poule pondeuse) ;

- Une mauvaise persistance de production d'oeufs ou de spermatozoïdes (20 à 25 
semaines au lieu de 50)

- L'existence de phases de repos d'origine saisonnière ou autre (ex. la couvaison).

A côté des progrès génétiques, on attend donc beaucoup des études des mécanismes neuroendocriniens sous-jacents à ces phénomènes pour tenter de les modifier ; ainsi la suppression de la rétroaction négative des stéroïdes sur l'hypothalamus des coqs ou l'inhibition de la prolactine des dindes constituent des voies de recherche prometteuses, mais la durée même des expérimentations (souvent un seul cycle par an) interdit d'espérer des progrès très rapides (de Reviers 1990).

On connaît par ailleurs les liaisons négatives existant entre le poids des coqs adultes et leur fertilité et, en conséquence, l'importance du rationnement alimentaire pour le maintien de leur production spermatique (de Reviers et Seigneurin 1990). Ce problème, joint à celui des besoins différentiels d'éclairement, conduit à envisager de plus en plus la séparation des deux sexes d'animaux reproducteurs afin de pouvoir appliquer à chacun, tant en phase juvénile qu'à l'age adulte, les conditions d'élevage permettant d'optimiser maturité sexuelle et persistance de production.

Cette situation rend évidemment obligatoire l'insémination artificielle dont l'usage en élevage industriel n'est encore systématique que pour la dinde, la pintade et, partiellement, pour la production de canards mulards. Cette technique est en revanche déjà utilisée pour les troupeaux de sélection de toutes les espèces, au moins pour les lignées "femelles». Certains sélectionneurs sont en effet encore réticents à utiliser l'I.A. au sein de lignées " mâles " dont les descendants auront à se reproduire par fécondation naturelle (Reddy et Sadjadi 1990). C'est là un des choix les plus importants à effectuer pour l'avenir, mais il est d'ores et déjà indispensable de développer d'une part une sélection des coqs et des poules en vue de leur utilisation en I.A., d'autre part des techniques de conservation du sperme à court et moyen terme qui permettent de gérer séparément les troupeaux adultes de mâles et de femelles.

\section{6 / Evolution de la pathologie et révolutions de la prophylaxie}

Dans le contexte des élevages hors sol, la définition même de la maladie a évolué « au point que toute anomalie ayant une incidence sur les performances zootechniques ..., toute contamination des produits obtenus (viande ou oeuf) par des micro-organismes affectant la santé humaine sont, à la limite, assimilées au fait pathologique, que la santé des animaux soit affectée ou non » (Bennejean 1990). Le champ de la «pathologie » aviaire s'en trouve élargi d'autant.

\section{1 / Des pathologies nouvelles}

A côté des grandes maladies aujourd'hui éradiquées (maladie de Newcastle, Pullorose ...) ou maîtrisées (coccidiose, maladies de Gumboro et

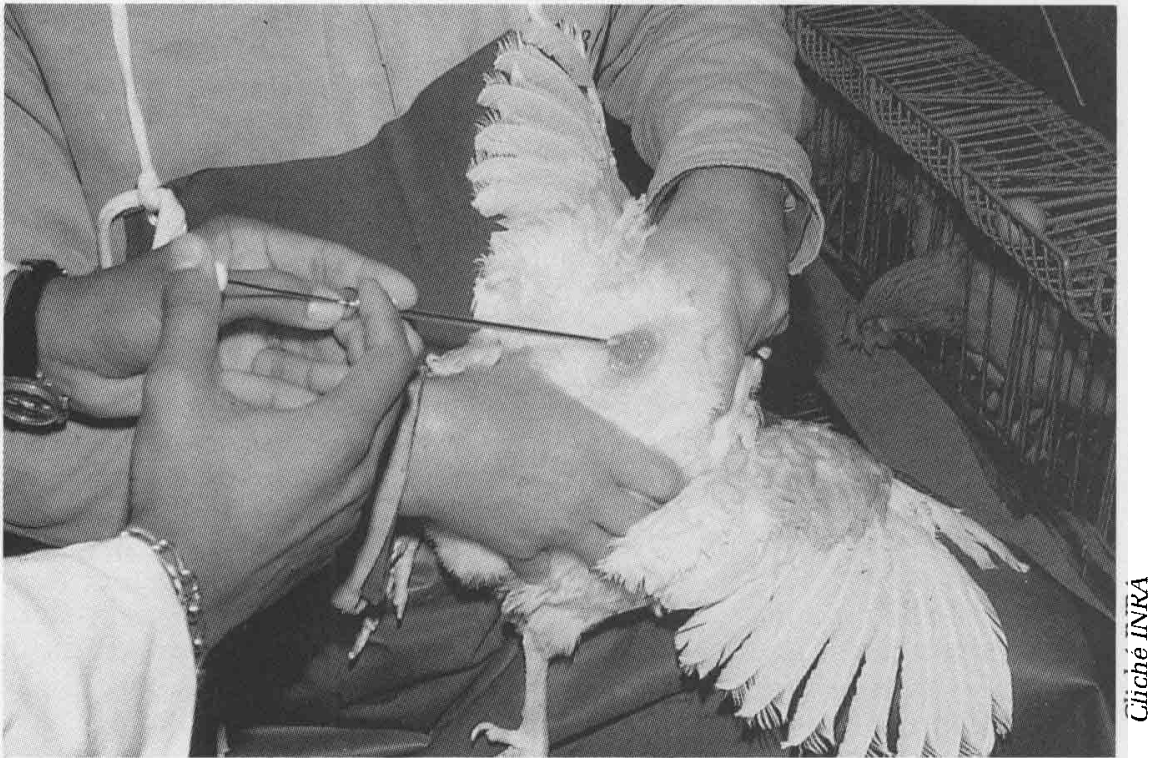

La généralisation de l'insémination artificielle, encore peu utilisée en élevage industriel pour l'espèce poule, permettra de gérer séparément les troupeaux de mâles et de femelles.

de Marek ...) se développent des maladies nouvelles telles que l'anémie infectieuse du poulet, le syndrome de la mort subite ou les troubles locomoteurs. Les stratégies à mettre en oeuvre face aux maladies infectieuses impliqueront des contrôles de plus en plus précis et rapides pour lesquels l'évolution des méthodes de diagnostic est fondamentale (voir ci-dessous paragraphe 6.5). La situation des autres maladies est sans doute plus complexe dans la mesure où elles paraissent souvent liées aux progrès réalisés sur la vitesse de croissance. Sans doute constituent-elles, dans l'état actuel des connaissances, le frein le plus important à ce progrès.

\section{2 / Le problème dit du " portage sain »}

L'épisode britannique des oeufs contaminés par Salmonella enteritidis en décembre 1988 a fait ressortir de façon exacerbée l'importance indubitable que tiendra la qualité microbiologique des produits dans les prochaines années. Le problème est complexe dans la mesure où les contaminations sont faibles (moins de 10 bactéries par oeuf et seulement 3 à 4 semaines après une contamination expérimentale) et où les animaux ne montrent aucun signe clinique.

Les stratégies de lutte doivent donc revêtir de nombreux aspects complémentaires comme cela a déjà été développé en Grande Bretagne ou par l'Union des Groupements de Producteurs d'oeufs de Bretagne, à savoir: contrôle systématique des couvoirs et des matières premières alimentaires (avec retrait des aliments contaminés) ; contrôle des poulos avant maturité sexuelle, ainsi qu'à 30 et 60 semaines d'âge et ... abattage éventuel des troupeaux infectés.

La mise en place d'un système d'assurancequalité tel que celui construit par le CIDEF (Comité Interprofessionnel de la Dinde Française) pour la viande de dinde relève de la 
même philosophie. Ces mesures draconiennes doivent par ailleurs s'accompagner de programmes de recherche portant sur la résistance génétique au portage (un modèle existe chez le mouton et la souris) et, encore plus rapidement, sur les méthodes de détection et de caractérisation des salmonelles (antigènes et sondes spécifiques de certains acides nucléiques).

D'autres moyens de lutte envisageables sont: - L'usage d'une flore de compétition qui pourrait au moins diminuer la multiplication de la bactérie. L'intervention in ovo n'est peut-être pas sans intérêt de ce point de vue (cf paragraphe 6.4 ci-dessous) ;

- L'administration de certains sucres (mannose et $\alpha$-méthyl $D$ mannoside) qui paraissent s'opposer à la fixation de la bactérie sur la paroi intestinale; l'excrétion fécale serait réduite d'un facteur 100 à 1000 (De Loach 1990).

\section{3 / Apparition de vaccins « recombinants »}

Avant que nous ne disposions d'animaux génétiquement résistants à certaines maladies, les vaccins vont être un terrain d'application privilégié des techniques de recombinaison génique. Ces techniques permettront par exemple de produire des vaccins dits de «sousunité » où les seules protéines immunogènes seront mises en œuvre, produites par des bactéries "recombinantes ». Un exemple de projet de ce type est développé en Australie pour la production d'un vaccin contre la maladie de Gumboro utilisant la protéine virale VP2 produite chez E. Coli et induisant, chez la poule, une réponse immunitaire protectrice du pous$\sin$.

Simultanément on cherche à développer des vaccins multipotents qui résulteraient par exemple de l'introduction chez un même virus porteur (lui-même neutralisé par le retrait d'un gène de virulence) de gènes codant pour des protéines immunogènes d'autres virus, voire de bactéries ou de parasites. Un tel montage est en cours avec le virus de la variole aviaire pour inclure une protection contre la Bronchite Infectieuse et la maladie de Newcastle (Anonyme 1990).

\section{4 / Mise en ouvre des vaccins}

Les avantages de tels vaccins et, plus tard, de véritables vaccins de synthèse, sont évidents: efficacité, sécurité, induction d'anticorps différents de ceux consécutifs à une infection, multipotence ... Tout vaccin avicole doit cependant posséder aussi les deux autres qualités indispensables que sont le faible prix de revient et la facilité d'administration. On soulignera de ce point de vue l'apparition de la vaccination in ovo qui a été présentée pour la première fois en 1989 pour un vaccin contre la maladie de Marek après que les capacités de réponse immunitaire de l'embryon aient fait l'objet de longs débats. Une automatisation des opérations de transfert en éclosoir, jointe à cette vaccination, assure le traitement de 20 à 30.000 œufs/heure/machine et permettra sans doute au procédé de s'imposer. On peut d'ailleurs penser à d'autres applications telles que l'administration in ovo de nutriments ou d'une flore spécifique.

\section{5 / Moyens de diagnostic}

Deux des plus importants outils de base de diagnostic sont dès aujourd'hui les anticorps monoclonaux et les tests ELISA (Enzyme Linked ImmunoSorbent Assay). Les premiers peuvent permettre par exemple de distinguer les souches vaccinales de virus de la bronchite infectieuse de ce qui est isolé chez des troupeaux lors de " passages » de la même maladie. L'introduction de tests ELISA comme outils précis et rapides de suivi sérologique peut permettre, quant à elle, le suivi des anticorps maternels transférés à la descendance ou celui d'un programme d'immunisation. Selon Shane (1990) les bénéfices issus des décisions qui pourront être prises à la suite de tels contrôles (traitements, vaccinations, changement de fournisseur de poussins ...) devraient couvrir environ 5 fois le coût de l'intervention.

Il n'en reste pas moins que, lorsque les taux de contamination et la réponse immunitaire sont très faibles (comme c'est le cas pour Salmonella enteritidis) la détection reste un problème majeur qui est encore loin d'être résolu.

\section{7 / De la technique à l'économie : satisfaire le consommateur}

Cette règle d'or n'est pas une nouveauté en aviculture. C'est précisément parce que les produits avicoles ont su répondre à l'évolution de la demande qu'ils ont connu une évolution soutenue au cours des années 80. Aujourd'hui cette satisfaction doit être comprise dans un sens de plus en plus large et s'appliquer non seulement aux caractéristiques du produit fini, mais également à la façon de l'obtenir en incluant les notions de protection des animaux et de l'environnement.

\section{1 / Evolution des produits}

La filière avicole française se caractérise par une très large diversification des produits qu'elle offre à différents segments de marchés bien identifiés. Cette diversification concerne : - les espèces produites (poulets, dindes, canards, pintades, etc ...) ;

- les modes de production (industriels ou de type « label»);

- les présentations des produits finis.

L'évolution récente de ce dernier point est particulièrement frappante. Ainsi, en 1980, $62 \%$ des poulets vendus en Grande-Bretagne étaient congelés et $38 \%$ présentés à l'état frais ; dix ans plus tard, la proportion est pratiquement inversée. Un autre exemple est fourni par l'évolution de la découpe des carcasses qui, à peu près inconnue en Europe il y a 10 ans (sauf pour la dinde), connaît une croissance comparable à celle observée aux Etats-Unis, illustrée par les chiffres du tableau 2. En outre, cette découpe ne constituera dans le futur qu'une première étape qui devra être suivie d'une 
Tableau 2. Evolution de la présentation des poulets aux USA (\% de la consommation totale).

\begin{tabular}{|c|c|c|c|}
\hline Année & Poulets entiers & Portions & $\begin{array}{c}\text { Préparations } \\
\text { élaborées }\end{array}$ \\
\hline 1963 & 81 & 17 & 2 \\
1989 & 20 & 59 & 21 \\
Prévision 2000 & 10 & 60 & 30 \\
\hline
\end{tabular}

seconde, voire d'une troisième tranformation aboutissant à des produits sans doute différents d'un pays à l'autre : hamburgers, nuggets, saucisses, viandes marinées, mousses ... puis plats cuisinés complets, tous produits pour lesquels salubrité et commodité seront les maître-mots de la qualité (à côté des critères de prix). Il convient donc bien désormais de considérer les viandes de volailles comme une matière première qui, selon Lescoat (1990, communication personnelle), " possède des qualités technologiques remarquables (pouvoir liant et émulsifiant) supérieures à celles de la viande de porc bien qu'avec une teneur en lipides plus faible ".

La forme de commercialisation des œufs connaît une évolution plus discrète mais néanmoins réelle : on estime ajourd'hui à $20 \%$ la part des œufs transformés en ovoproduits aux USA avec une progression relative de $22 \%$ au cours des cinq dernières années. La nature des ovoproduits fabriqués varie également (tableau 3), au profit de ceux « frais pasteurisés " et " poudre » offrant des garanties microbiologiques supérieures à celles des ovoproduits congelés. Un autre avantage dont disposeront sans doute les ovoproduits futurs est de pouvoir surmonter le handicap du cholestérol (cf paragraphe suivant) grâce à des procédés d'extraction encore trop coûteux aujourd'hui. Enfin, la préparation, pour les collectivités, d'œufs durs écoqués et d'omelettes connaît en France un succès croissant.

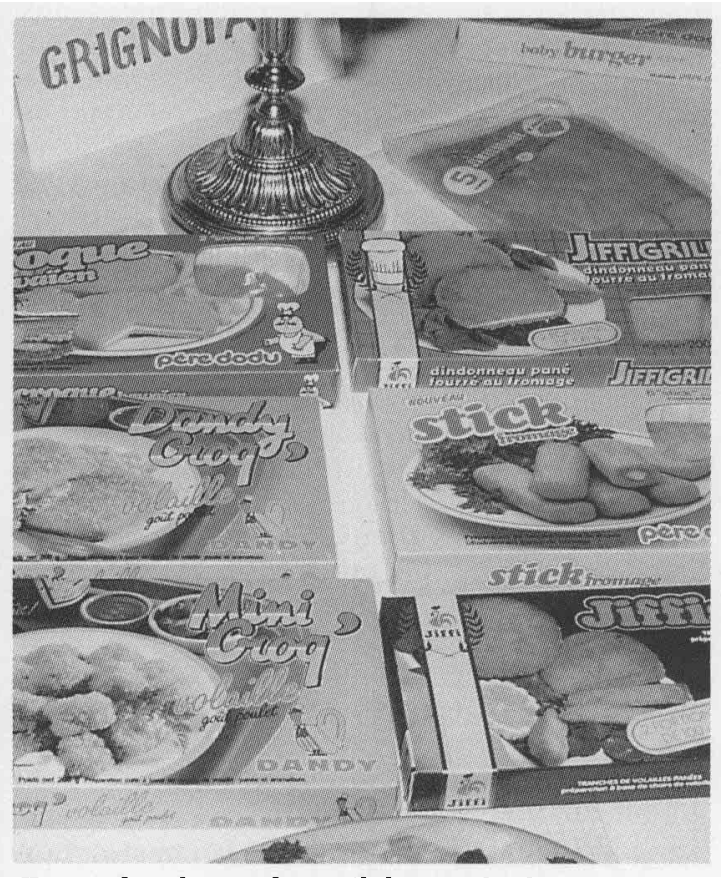

Exemples de produits élaborés de dinde.

\section{2 / Prise en compte des soucis nutritionnels}

Naber (1990) note à juste titre que nous sommes entrés dans une phase de « cholestérolophobie» irraisonnée qui risque de porter à l'œuf un préjudice injustifié. La première précaution à prendre est donc d'afficher des teneurs de l'œuf en cholestérol qui ne soient pas surévaluées: depuis qu'aux USA les teneurs moyennes publiées ont été, à juste titre, abaissées, la recommandation de consommation de l'American Heart Association est passée de 3 à 4 œufs/semaine, soit une augmentation de $33 \%$ ! Pour le reste, aucune voie d'action

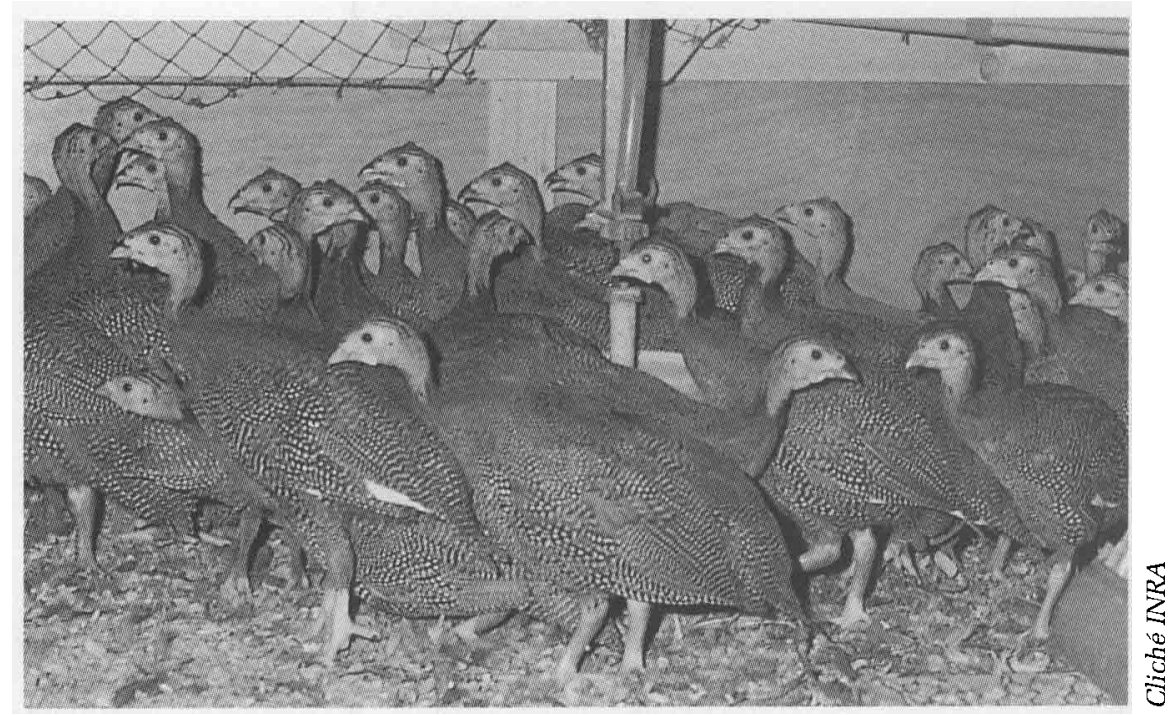

Pintadeaux en croissance.

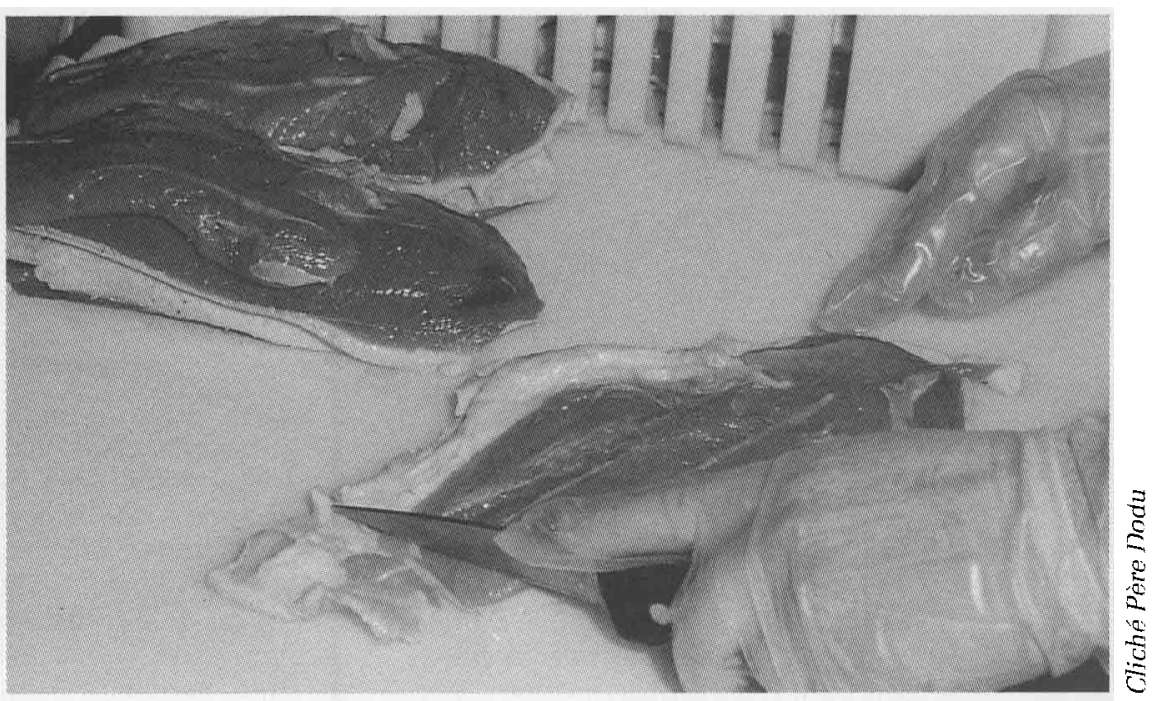

Filets de canards de Barbarie. 
Tableau 3. Evolution de la présentation des ovoproduits aux USA (en \% du total).

\begin{tabular}{|c|c|c|c|}
\hline Année & $\begin{array}{c}\text { Frais } \\
\text { Pasteurisés }\end{array}$ & Congelés & Poudre \\
\hline 1952 & 3 & 84 & 13 \\
1987 & 30 & 34 & 36 \\
\hline
\end{tabular}

génétique ou nutritionnelle pour réduire, via la poule, la teneur de l'œuf en cholestérol ne paraît pouvoir être retenue de façon sérieuse. A l'inverse les teneurs du jaune en acides gras poly-insaturés ou en vitamines liposolubles pourraient être facilement augmentées.

Un autre secteur sensible est celui des additifs (antibiotiques, anticoccidiens, antioxydants...) dont il n'est pas exclu que le consommateur souhaite voir disparaître toute trace dans certains produits « hauts de gamme » de demain. Le développement d'animaux génétiquement résistants à des maladies sera, de ce point de vue, un atout positif.

\section{3 / Vers une éthique de production}

Les objections éthiques à la production animale intensive englobent à la fois les problèmes liés au confinement ou à certaines manipulations des animaux et des aspects plus indirects tels que les éventuelles répercussions sur la santé animale et humaine (Lindgren 1976). Une attitude positive face à cette « demande " semble être :

- d'écouter la critique et de développer des études qui permettent d'apprécier objectivement les bases du "bien-être » animal; en ce domaine, l'INRA conduit depuis quinze ans un programme de recherche sur les " besoins en environnement » de la poule ;

- en s'appuyant sur des résultats concrets, de multiplier les occasions d'éducation d'un public urbanisé qui, tout en vivant loin de la production animale, pense savoir, à partir de raisonnements anthropomorphiques, comment élever des animaux ;

- d'élaborer des règlements internationaux qui, tout en tenant compte du bien-être animal, permettent de maintenir une juste concurrence entre pays producteurs.

\section{8 / Des stratégies de production et de commercialisation}

Toute réflexion sur l'organisation d'une production doit prendre en compte des exigences aussi diverses que l'approvisionnement et le coût des intrants (aliments, énergie ...), la protection des animaux et de l'environnement, l'existence ou non de débouchés locaux ... et l'assurance d'un profit suffisant pour chaque acteur de la filière. C'est donc une démarche difficile dont seuls quelques éléments sont apportés ici à titre d'exemples.

\section{1 / Où et comment produire ?}

Les années 70 ont vu s'implanter un grand nombre de bâtiments clos à ventilation dynamique pour la production de poulets. Des poulaillers dits « ouverts » se développent en Bretagne depuis quelques années : leur coût d'investissements est plus faible et l'utilisation de l'espace par les animaux est meilleure, au moins durant les premières semaines. Cette évolution, réservée, il est vrai, à des conditions climatiques spécifiques, montre que des solutions traditionnelles d'élevage peuvent être ré-actualisées et permettre des performances commerciales satisfaisantes.

Dans le même contexte s'est développé l'usage d'une litière dite " accumulée» qui apporte certainement des éléments de confort, mais pourrait être une source de transmission horizontale de certains microorganismes. L'assurance-qualité voulue dans le futur devra précisément estimer l'impact de chacun de ces points.

Les grands élevages de poules pondeuses d'œufs de consommation gagneraient, quant à eux, à travailler de plus en plus avec des bandes d'animaux d'âge unique, mais ceci conduira nécessairement à spécialiser aussi les circuits de distribution des œufs. La stratégie des «fermes de ponte» utilisant leurs propres céréales est un modèle intéressant malgré les limitations inhérentes au système concernant les marges possibles d'optimisation des formules alimentaires et l'impossibilité d'appliquer certains traitements technologiques aux aliments. Le retour à l'élevage de poules au sol est également une alternative possible pour une partie de la production à condition que, là encore, la qualité bactériologique de l'œuf obtenu soit rigoureusement surveillée (Sauveur 1991).

\section{2 / Prise en compte de résultats économiques}

Bien que, comme cela était rappelé en introduction, l'aviculture française bénéficie d'une organisation en filière structurée, il ne semble pas que la pratique d'une gestion par résultats économiques vrais prenant en compte la totalité d'une chaîne de production ait été suffisamment développée à ce jour. Il est clair par exemple que l'attention longtemps maintenue sur l'indice de consommation comme critère principal d'efficacité en production de viande est le fait d'une gestion parcellisée. Des modèles d'analyse plus globaux seraient aujourd'hui bienvenus.

Un exemple en est donné par la construction de Belyavin (1990) appliquée à la poule pon- 
deuse qui tente de guider les choix concernant trois facteurs d'élevage des poulettes et deux facteurs d'élevage des poules adultes en fonction du prix de l'aliment et de l'œuf (voir tableau 4).

Un autre exemple typique est celui de la taille optimale que devra avoir le poulet de demain. Une réponse souvent entendue est qu'il devra être le plus gros possible $\{3,5 \mathrm{~kg}$ par exemple) pour permettre une découpe automatique de morceaux d'épaisseur constante dont le coût serait diminué de 25 à $50 \%$. Une étude récemment conduite en Géorgie intégrant l'indice de consommation, la mortalité, le tăux de déclassement et le coût d'abattage de poulets pesant $1,6,2,3$ ou $2,9 \mathrm{~kg}$ montre cependant que le coût de l'unité de poids vif s'accroît de $30 \%$ entre le plus léger et le plus lourd. Qu'en seraitil à 3,5 kg ? Et si l'on intégrait dans le calcul le coût du logement, le rendement d'abattage, etc... ? Il paraît plus prudent d'affirmer que l'optimum économique reste aujourd'hui à déterminer.

\section{3 / Les stratégies commerciales d'appui}

Deux stratégies strictement commerciales, parmi d'autres, sont intéressantes à rapporter ici : la prise en compte de « l'image qualité » et le développement des marques.

"L'image-qualité » met l'accent sur la qualité telle qu'elle est perçue et non telle qu'elle existe intrinsèquement (Panigyrakis 1986). Elle intègre donc le fait que les sentiments du consommateur l'emportent sur la logique, que les propriétés technologiques réelles du produit sont peu comprises et que l'acceptation sociale d'un produit modifie la perception de sa qualité. «Pour ces raisons, un changement de qualité peut être réel ou imaginaire ... Un changement léger de qualité combiné à une importante publicité peut être beaucoup plus profitable que l'inverse ».

Le développement d'une politique de marques et de marketing a permis qu'en France la consommation d'œufs continue à croître entre 1982 et 1987 alors qu'elle diminuait dans tous les pays développés. Plusieurs auteurs estiment que la politique de marque est également profitable pour le poulet et qu'elle permettra de préserver, dans certains créneaux, des marges relativement stables. Elle est en tous cas cohérente avec le développement des différentes formes de certification qui devraient se mettre en place au cours des prochaines années.

En conclusion, l'aviculture peut être définie comme un ensemble d'industries appliquées à des êtres vivants complexes, les oiseaux domestiques. Son évolution est donc guidée à la fois par les motivations financières des groupes industriels et par les révolutions que connaissent les sciences biologiques. C'est l'interaction de ces deux courants qui assure le dynamisme d'une filière aujourd'hui enviée. Peut-être doiton cependant réfléchir à d'éventuelles évolu-

Tableau 4. Modèle théorique de choix entre différentes alternatives d'élevage des poulettes et des poules pondeuses selon les prix d'achat de l'aliment et de vente de l'œuf (Belyavin 1990).

Les alternatives d'élevage envisagées sont les suivantes :

- pour les poulettes alimentation : AL : ad libitum $\mathrm{R}$ : restreinte programme lumineux : $\quad \mathrm{C}$ : conventionnel (abaissement à $8 \mathrm{~h} /$ jour en fin de $1^{\text {te }}$ semaine), $\mathrm{Pr}$ : progressif (abaissement à $8 \mathrm{~h} / \mathrm{jour}$ étalé sur 8 semaines)

- pour les poules en ponte aliments à concentration : $\quad \mathrm{H}$ : haute $(18 \% \mathrm{MAT}, 11,7 \mathrm{MJ} / \mathrm{kg}), \mathrm{M}$ : moyenne, B : basse $(15,5 \% \mathrm{MAT}, 10,8 \mathrm{MJ} / \mathrm{kg})$

programme lumineux : $\quad \mathrm{N}$ : normal, Fr : fractionné en blocs réguliers

\begin{tabular}{|c|c|c|c|c|c|c|}
\hline & & & \multicolumn{4}{|c|}{ Prix de l'œuf } \\
\hline & & & \multicolumn{2}{|c|}{ Haut } & \multicolumn{2}{|c|}{ Bas } \\
\hline & & & $\begin{array}{l}\text { Ecarts } \\
\text { larges }\end{array}$ & $\begin{array}{l}\text { Ecarts } \\
\text { faibles }\end{array}$ & $\begin{array}{l}\text { Ecarts } \\
\text { larges }\end{array}$ & $\begin{array}{l}\text { Ecarts } \\
\text { faibles }\end{array}$ \\
\hline \multirow{4}{*}{$\begin{array}{c}\text { Prix } \\
\text { de } \\
\text { l'aliment }\end{array}$} & \multirow{2}{*}{ Haut } & $\begin{array}{l}\text { Ecarts } \\
\text { larges }\end{array}$ & $\begin{array}{l}1 \\
\text { AL. Pr } \\
\text { M. Fr }\end{array}$ & $\begin{array}{l}5 \\
\text { Al. C } \\
\text { M. N }\end{array}$ & $\begin{array}{l}9 \\
\text { R. } \mathrm{Pr} \\
\text { B. } \mathrm{Fr}\end{array}$ & $\begin{array}{l}13 \\
\text { R. C } \\
\text { B. } \mathrm{N}\end{array}$ \\
\hline & & $\begin{array}{l}\text { Ecarts } \\
\text { faibles }\end{array}$ & $\begin{array}{l}2 \\
\text { Al. Pr } \\
\text { M/H Fr }\end{array}$ & $\begin{array}{l}6 \\
\text { AI. C } \\
\text { M. N }\end{array}$ & $\begin{array}{l}10 \\
\text { R. Pr } \\
\text { M. Fr }\end{array}$ & $\begin{array}{l}14 \\
\text { R. C. } \\
\text { B. N. }\end{array}$ \\
\hline & \multirow{2}{*}{ Bas } & $\begin{array}{l}\text { Ecarts } \\
\text { larges }\end{array}$ & $\begin{array}{l}3 \\
\text { Al. Pr } \\
\text { M/H Fr }\end{array}$ & $\begin{array}{l}7 \\
\text { Al. C } \\
\text { M. N }\end{array}$ & $\begin{array}{l}11 \\
\quad \text { R. Pr } \\
\text { M. Fr }\end{array}$ & $\begin{array}{l}15 \\
\text { R. C } \\
\text { M. N }\end{array}$ \\
\hline & & $\begin{array}{l}\text { Ecarts } \\
\text { faibles }\end{array}$ & $\begin{array}{l}4 \\
\text { Al. Pr } \\
\text { H. N }\end{array}$ & $\begin{array}{l}8 \\
\text { Al. C } \\
\text { H. N }\end{array}$ & $\begin{array}{l}12 \\
\text { R. Pr } \\
M / H \text { Fr/N }\end{array}$ & $\begin{array}{l}16 \\
\text { R. C } \\
\text { H. N }\end{array}$ \\
\hline
\end{tabular}

16 solutions sont proposées selon que les prix d'aliment et d'œufs sont hauts ou bas et selon que les plages de variation de ces prix sont importantes ou non (on considère comme " importante " une variation de prix de plus de 5-6\% entre 2 aliments $\mathrm{H}$ et $\mathrm{B}$ et une variation de prix de plus de 5 c. entre 2 cufs de calibre 1 et 3$)$. 
tions structurelles qui lui permettraient de mieux intégrer encore l'ensemble des progrès techniques dans les choix économiques de production.

\section{Remerciements}

L'auteur adresse ses remerciements à Madame Catherine Beaumont dont les notes de lecture ont été très appréciées.

\section{Principales références bibliographiques}

La liste complète peut être obtenue auprès de l'auteur.

ANONYME, 1990. Australian biotechnology challenges disease. Poult. Int., 29(6), 14-16.

BELYAVIN C., 1990. Flexible approach to managing layers. Poult. Int., 29(6), 18-22.

BENNEJEAN G., 1990. Les contraintes sanitaires en aviculture. C.R. Acad. Agric. Fr., 76, sous presse

BLUM J.C., 1990. Les aliments et l'alimentation. C.R. Acad. Agric. Fr., 76, sous presse.

BULFIELD G., MC KAY J.C., 1987. Genetic manipulation of egg quality. In : «Egg quality - Current problems and recent advances» (Wells R.G., Belyavin C.G. ed.) Butterworths (Seven oaks), 195-200.

DE LOACH J.R., 1990. Salmonella prevention with carbohydrates. Poult. Int., 29(4), 36-37.

FREEMAN B.M., BUMSTEAD N., 1987. Transgenic poultry : theory and practice. World's Poult. Sci. J., 43, 180189.

JOHNSON R.J., 1989. Growth physiology and biotechnology: potential to improve broiler production. World's Poult. Sci. J., 46, 228-240.
KUHLEIN U., ZADWORNY D., 1990. Molecular aspects of poultry breeding. 4th World Cong. Genet. Appl. Livest. Prod., Edinburgh, XVI, 21-30.

LECLERCQ B., 1989. Possibilités d'obtention et intérêt des génotypes maigres en aviculture. INRA Prod. Anim., $2,275-286$

LINDGREN N.O., 1976. The conflict between technica advances and ethics in animal production. World's Poult. Sci. J., 32, 243-256.

MALLARD J., DOUAIRE M., 1990. Evolution de la sélection avicole. C.R. Acad. Agric. Fr., 76, sous presse

NABER E.C., 1990. Cholesterol content of eggs : can and should it be changed ? Feedstuffs, 62(5), 46-52.

NORDSKOG A.W., 1983. Immunogenetics as an aid to selection for disease resistance in the fowl. World's Poult. Sci. J., 39, 199-209.

PANIGYRAKIS G., 1986. La qualité perçue et le comportement d'achat. Econom. Gest. Agro. Alim., 2, 28-34.

PICARD M., 1990. Des recherches pour lever les contraintes de l'alimentation des volailles. L'Aviculteur, $513,49-51$

REDDY R.P., SADIADI M., 1990. Selection for growth and semen traits in the poultry industry: what can we expect in the future. In: "Control of Fertility in Domestic Birds ", Les Colloques de l'INRA $n^{\circ} 54$, INRA Ed., Paris, 47-60.

De REVIERS M. 1990. La reproduction des oiseaux domestiques. Quelles chances pour l'avenir? C.R. Acad Agric. Fr., 76, sous presse.

SAUVEUR B., 1991. Effets du mode de production sur les caractéristiques de l'œuf. Mise au point bibliographique INRA Prod. Anim., 4, sous presse.

SHANE S.M., 1990. Elisa technology and flock health. Poult. Int., 29(4), 24-26.

SIEGEL P.B., 1990. Poultry breeding, 4th World Cong. Genet. Appl. Livest. Prod., Edinburgh, XVI, 3-4

VAN DEN EYDEN G.P.A., 1979. La génétique et l'industrie du broiler en l'an 2000. NRA Bull., Sept-Oct. 1979.

\section{Summary}

Strategies for further technical and economic progress in poultry production.

The combination of scientific, technic and economic arguments can still contribute to the elaboration of numerous progress strategies in poultry production. Thus, improvements induced by classical quantitative genetics are far from being ended. New selection criteria are in progress (both in anatomy and physiology). These can be complemented by studies of DNA polymorphisms used as predictors of economic characters such as resistance to illness. However these methods are expensive such as trangenesis which is still limited to the methodological study stage. These methods will be applied practically only if the feasibility to increase an economic trait is clearly demonstrated. In the meantime, any somatotropin induced growth stimulation seems to be excluded.

The metabolic utilization of feedstuffs will require an evaluation which is increasingly precise. It may be sometimes enhanced by enzymes in order to decrease environment pollution (nitrogen and phosphorus). The achievement of safe (without pathogenic bacteria) complete feed will be also required. The future of reproduction depends on the control of the breeders' feed intake and, later, on a general use of artificial insemination which will allow a totally separated management of the two sexes.

Poultry pathology is diversifying on account of new complex syndromes such as infectious runting or leg weakness. The animal transport of pathogenic (for man) bacteria will be the other main concern henceforth. Multivalent vaccines can be prepared by gene recombination techniques which will place side by side genes of different antigenic sites inside a bacteria or a virus. An other innovation may be the administration in ovo of some vaccines during the egg transfer to the hatcher.

Rearing techniques have still to be adapted to the specificities of each country and even of each area; they must take first into account the bacteriological quality of the final products and the animal welfare problem. Poultry products will be increasingly submitted to a first, and even to a second transformation which will continually increase their practicability and dietetic value. They will be promoted by trade-mark and advertising politics. As a whole, economic optimization studies integrating all the operations of a network production have still to be developed

SAUVEUR B., 1991. Stratégies pour de nouveaux progrès techniques et économiques en aviculture. INRA Prod. Anim., 4 (1), 31-40. 